\title{
Lower Bounds for Order of Decay or of Growthin Time for Solutions to Linear and Non-linear Schrödinger Equations
}

\author{
By \\ Tohru OzAWA* and Nakao HaYASH ${ }^{* *}$
}

\begin{abstract}
We study lower bounds of decay (or of growth) order in time for solutions to the Cauchy problem for the Schrödinger equation:

$$
\begin{aligned}
& i \partial_{t} u=-\Delta u+f(u),(t, x) \in \mathbb{R} \times \mathbb{R}^{n} \quad(n \geq 1), \\
& u(0)=\phi, \quad x \in \mathbb{R}^{n},
\end{aligned}
$$

where $f$ is a linear or non-linear complex-valued function.

Under some conditions on $f$ and $\phi$, it is shown that every nontrivial solution $u$ has the estimate

$$
\liminf _{t \rightarrow \pm \infty}|t| n / 2-n / q\|u(t)\|_{L^{q}(|x|<k|t|)}>0
$$

for sufficiently large $k>0$ and for any $q \in[2, \infty]$.

In the previous work [12] of the first named author, we imposed on the assumption that $u$ is asymptotically free. In this article, however, we shall show the assumption is, in fact, irrelevant to the results.
\end{abstract}

\section{§1. Introduction}

In this paper we consider the asymptotic behavior in time of solutions to the equation:

$$
\left\{\begin{array}{l}
i \partial_{t} u=-\Delta u+f(u),(t, x) \in \mathbb{R} \times \mathbb{R}^{n} \quad(n \geq 1), \\
u(0)=\phi \neq 0, \quad x \in \mathbb{R}^{n},
\end{array}\right.
$$

where $f$ describes a linear or non-linear perturbation and $\phi$ is a given initial data.

Communicated by S. Matsuura, April 4, 1988.

* Department of Mathematics, Nagoya University, Nagoya 464, Japan.

** Department of Mathematics, Faculty of Engineering, Gunma University, Kiryu 376, Japan. 
More precisely, we deal with the following three types of equations.

(1) Non-linear Schrödinger equation with power interaction:

$$
i \partial_{t} u=-\Delta u+\lambda|u|^{p-1} u,
$$

where $\lambda>0$ and $1<p<\alpha(n)$ with $\alpha(n)=\infty(n=1,2), \alpha(n)=(n+2) /(n-2)(n \geq 3)$.

(2) Non-linear Schrödinger equation with non-local interaction:

$$
i \partial_{t} u=-\Delta u+\left(V *|u|^{2}\right) u,
$$

where $V=V(x)=\lambda|x|^{-\gamma}$ with $\lambda>0$ and $0<\gamma<\min (4, n)$.

(3) Linear Schrödinger equation with long-range perturbation:

$$
i \partial_{t} u=H u, \quad H=H_{0}+V,
$$

where $H_{0}$ is the self-adjoint realization of $-\Delta$ in the Hilbert space $L^{2}=L^{2}\left(\mathbb{R}^{n}\right)$ and the long-range perturbation $V$ is assumed to satisfy some conditions specified later.

Concerning the asymptotic behavior in time of a solution $u$ for (1.1), it is possible to distinguish between the following two cases (a) and (b) in the category of $L^{2}$-scattering theories.

(a) There exist some (or equivalently, unique) states $u_{ \pm} \in L^{2}$ such that

$$
\lim _{t \rightarrow \pm \infty}\left\|u(t)-e^{-i t H_{0}} u_{ \pm}\right\|_{L^{2}}=0,
$$

where $\left\{e^{-i t H_{0}} ; t \in \mathbb{R}\right\}$ is the free Schrödinger evolution group. In this case, we call a solution $u$ asymptotically free.

(b) There do not exist any states $u_{ \pm} \in L^{2}$ satisfying (1.2).

In the case (a), it has been proved in [1] that every non-trivial solution $u$ has the estimate

$$
\liminf _{t \rightarrow \pm \infty}|t|^{n / 2-n / q}\|i(t)\|_{L^{q}\left(k^{\prime}|t|<|x|<k|t|\right)}>0
$$

for some $0<k^{\prime}<k$ and for any $q \in[2, \infty]$. In view of the proof, it is clear that $k$ and $k^{\prime}$ in (1.3) depend on the momentum support of $u_{ \pm}$, i.e., the support of the Fourier transform. Therefore, the argument in [12] only gives rather implicit relations between the pair $\left(k^{\prime}, k\right)$ and the initial data $\phi$.

We now state our main purpose in this paper, which is twofold.

One is to obtain similar lower-bound estimates as in (1.3) even when (1.2) 
do not hold. To be more specific, we show that the following estimate slightly weaker than (1.3)

$$
\liminf _{t \rightarrow \pm \infty}|t|^{n / 2-n / q}|| u(t) \|_{L^{q}(|x|<k|t|)}>0
$$

holds for non-trivial solutions to the equations (1)-(3).

The other is to give explicit lower bounds of $k$ in (1.4) in terms of the given initial data $\phi$.

As corollaries to main theorems proved in this paper, we see that $L^{p}$-decay or growth estimates obtained by many peoples (see [1], [3], [4], [7], [8], [10] and [11]) are optimal.

Finally we list some notations which will be used in the sequel.

$\sum$ denotes the Hilbert space

$$
\Sigma=\left\{u \in L^{2}\left(\boldsymbol{R}^{n}\right) ; \partial_{j} u, x_{j} u \in L^{2}\left(\mathbb{R}^{n}\right) \quad(j=1, \cdots, n)\right\}
$$

with the norm

$$
\|u\|_{\Sigma}=\left(\|u\|_{2}^{2}+\sum_{j=1}^{n}\left\|\partial_{j} u\right\|_{2}^{2}+\sum_{j=1}^{n}\left\|x_{j} u\right\|_{2}^{2}\right)^{1 / 2} .
$$

$\|\cdot\|_{p}$ denotes the usual $L^{p}\left(\mathbb{R}^{n}\right)$-norm and $(\cdot, \cdot)$ denotes the $L^{2}\left(\boldsymbol{R}^{n}\right)$-scalar product. $H^{1}\left(\boldsymbol{R}^{n}\right)$ denotes the usual Sobolev space of order one. For an interval $I \subset \mathbb{R}$ and a Banach space $E, C(I ; E)$ denotes the space consisting of $E$-valued continuous functions on $I$ and $\|\cdot\|_{\mathcal{L}(E)}$ denotes the operator norm on the space of all bounded linear maps from $E$ into $E$. For a self-adjoint operator $H$ in the Hilbert space $L^{2}\left(\mathbb{R}^{n}\right), \mathcal{H}_{\text {cont }}(H)$ denotes the continuous spectral subspace of $H$. $-i \nabla$ and $x$ also denote the momentum operator and the position operator acting on the Hilbert space $L^{2}\left(\mathbb{R}^{n}\right) \otimes C^{n}$, respectively. Different positive constants might be denoted by the same letter $C$, if necessary, by $C(*, \cdots, *)$ in order to indicate constants depending only on the quantities appearing in parentheses.

\section{§ 2. Non-linear Schrödinger Equations with Power Interaction}

In this section we consider the equation of the form:

$$
\left\{\begin{array}{l}
i \partial_{t} u=-\Delta u+|u|^{p-1} u,(t, x) \in \boldsymbol{R} \times \boldsymbol{R}^{n} \quad(n \geq 1), \\
u(0)=\phi, \quad x \in \mathbb{R}^{n},
\end{array}\right.
$$

where $1<p<\alpha(n)$. By a mild solution of (2.1), we mean a function $u \in$ $C\left(\mathbb{R} ; L^{2}\right)$ satisfying the integral equation 


$$
u(t)=e^{-i t H_{0}} \phi-i \int_{0}^{t} e^{-i(t-\tau) H_{0}}\left(|u(\tau)|^{p-1} u(\tau)\right) d \tau \quad \text { in } \quad L^{2}
$$

for any $t \in \mathbb{R}$. We summarize the results concerning mild solutions of (2.1).

Lemma 1. Let $\phi \in \Sigma$. Then there exists a unique mild solution $u \in$ $C\left(\mathbb{R} ; \sum\right)$ of (2.1) satisfying

$$
\|u(t)\|_{2}=-\|\phi\|_{2}
$$

and

$$
\|\nabla u(t)\|_{2}^{2}+\frac{2}{p+1}\|u(t)\|_{p+1}^{p+1}=\|\nabla \phi\|_{2}^{2}+\frac{2}{p+1}\|\phi\|_{p+1}^{p+1}
$$

for $t \in \mathbb{R}$. Furthermore, $u$ satisfies

$$
\begin{aligned}
& \|(x+2 i t \nabla) u(t)\|_{2}^{2}+\frac{8}{p+1} t^{2}\|u(t)\|_{p+1}^{p+1} \\
& =\|(x+2 i s \nabla) u(s)\|_{2}^{2}+\frac{8}{p+1} s^{2}\|u(s)\|_{p+1}^{p+1} \\
& \quad+\frac{4(n+4-n p)}{p+1} \int_{s}^{t} \tau\|u(\tau)\|_{p+1}^{p+1} d \tau, \quad t, s \in \mathbb{R}, \\
& \|u(t)\|_{p+1} \leq C\left(n, p,\|\phi\|_{\Sigma}\right) \cdot(1+|t|)^{-\theta(p)}, \quad t \in \mathbb{R},
\end{aligned}
$$

and

$$
\|(x+2 i t \nabla) u(t)\|_{2} \leq C\left(n, p,\|\phi\|_{\Sigma}\right) \cdot(1+|t|)^{a(p)}, \quad t \in \mathbb{R},
$$

where $\theta(p)=n(p-1) / 2(p+1)$ and $a(p)=1-n(p-1) / 4$ if $1<p \leq r(n)=(n+2+$ $\left.\left(n^{2}+12 n+4\right)^{1 / 2}\right) / 2 n, a(p)=0$ if $r(n)<p<\alpha(n)$.

For Lemma 1, see, e.g., [1], [3] and [15].

We now have:

Theorem 耳. Let $\phi \in \Sigma \backslash\{0\}$ and let $u \in C(\mathbb{R} ; \Sigma)$ be the solution given by Lemma 1. Then for any

$$
k>k_{0}=2\left(\|\nabla \phi\|_{2}^{2}+\frac{2}{p+1}\|\phi\|_{p+1}^{p+1}\right)^{1 / 2} /\|\phi\|_{2},
$$

we have

$$
\liminf _{t \rightarrow \pm \infty} \int_{|x|<k|t|}|u(t, x)|^{2} d x>0 .
$$

Proof. We assume 


$$
\liminf _{t \rightarrow \infty} \int_{|x|<k t}|u(t, x)|^{2} d x=0
$$

for some $k>k_{0}$ and we deduce a contradiction. From the assumption (2.10), there exist a sequence $\left\{t_{j} ; j \geq 1\right\}$ in $\boldsymbol{R}$ such that $0<t_{1}<t_{2}<\cdots<t_{j} \uparrow \infty$ as $j \rightarrow \infty$ and

$$
\lim _{j \rightarrow \infty} \int_{|x|<k t_{j}}\left|u\left(t_{j}, x\right)\right|^{2} d x=0
$$

(2.7) and (2.11) give

$$
\begin{aligned}
& \int_{|x|<k t_{j}}\left|\nabla u\left(t_{j}, x\right)\right|^{2} d x \\
& \quad \leq 2 \int_{|x|<k t_{j}}\left|\frac{x}{2 i t_{j}} u\left(t_{j}, x\right)\right|^{2} d x+2 \int_{|x|<k t_{j}}\left|\left(\frac{x}{2 i t_{j}}+\nabla\right) u\left(t_{j}, x\right)\right|^{2} d x \\
& \quad \leq \frac{k^{2}}{2} \int_{|x|<k t_{j}}\left|u\left(t_{j}, x\right)\right|^{2} d x+2 \|\left.\left(\frac{x}{2 i t_{j}}+\nabla\right) u\left(t_{j}\right)\right|_{2} ^{2} \rightarrow 0 \quad(j \rightarrow \infty),
\end{aligned}
$$

from which we get

$$
\begin{aligned}
\lim _{j \rightarrow \infty} & \int_{|x|>k t_{j}}\left|\nabla u\left(t_{j}, x\right)\right|^{2} d x \\
& =\lim _{j \rightarrow \infty}\left\|\nabla u\left(t_{j}\right)\right\|_{2}^{2} \\
& =\lim _{j \rightarrow \infty}\left(\|\nabla \phi\|_{2}^{2}+\frac{2}{p+1}\|\phi\|_{p+1}^{p+1}-\frac{2}{p+1}\left\|u\left(t_{j}\right)\right\|_{p+1}^{p+1}\right) \\
& =\|\nabla \phi\|_{2}^{2}+\frac{2}{p+1}\|\phi\|_{p+1}^{p+1} .
\end{aligned}
$$

Here we have used (2.4) and (2.6). Similarly, by (2.3) and (2.11) we have

$$
\lim _{j \rightarrow \infty} \int_{|x|>k t_{j}}\left|u\left(t_{j}, x\right)\right|^{2} d x=\|\phi\|_{2}^{2}
$$

A simple calculation leads to

$$
\begin{aligned}
& \left(\int_{|x|>k t_{j}}\left|\nabla u\left(t_{j}, x\right)\right|^{2} d x\right)^{1 / 2} \\
& \quad \geq \frac{k}{2}\left(\int_{|x|>k t_{j}}\left|u\left(t_{j}, x\right)\right|^{2} d x\right)^{1 / 2}-\left\|\left(\frac{x}{2 i t_{j}}+\nabla\right) u\left(t_{j}\right)\right\|_{2} .
\end{aligned}
$$

We take the limit $j \rightarrow \infty$ in (2.14) and apply (2.12)-(2.13) to (2.14) to conclude

$$
\left(\|\nabla \phi\|_{2}^{2}+\frac{2}{p+1}\|\phi\|_{p+1}^{p+1}\right)^{1 / 2} \geq \frac{k}{2}\|\phi\|_{2} .
$$


This contradicts the fact that $k>k_{0}$.

The case $r<0$ can be treated similarly.

Q.E.D.

Remark 1. (2.9) gives a propagation property of quantum particles obeying non-linear Schrödinger equations with power interaction. We also have from (2.6) that for any $R>0$,

$$
\lim _{t \rightarrow \pm \infty} \int_{|x|<R}|u(t, x)|^{2} d x=0
$$

Compare (2.9).

Corollary 1. Under the assumptions of Theorem 1, the unique mild solution $u \in C\left(\mathbb{R} ; \sum\right)$ has the estimate

$$
\liminf _{t \rightarrow \pm \infty}|t|^{n / 2-n / q}|| u(t) \|_{L^{q}(|x|<k|t|)}>0
$$

for any $k>k_{0}$ and $q \in[2, \infty]$.

Proof. (2.15) is an easy consequence of (2.9) and the Hölder inequality.

Q.E.D.

Remark 2. Lower-bound estimates for the case $1+2 / n<p<\alpha(n)$ have been obtained in [12].

\section{§3. Non-linear Schnödinger Equations with Non-local Interaction}

This section deals with the following Hartree type equation:

$$
\left\{\begin{array}{l}
i \partial_{t} u=-\Delta u+\left(V *|u|^{2}\right) u,(t, x) \in \mathbb{R} \times \mathbb{R}^{n} \quad(n \geq 1), \\
u(0)=\phi, \quad x \in \mathbb{R}^{n},
\end{array}\right.
$$

where $V=V(x)=|x|^{-\gamma}$ with $0<\gamma<\min (4, n)$.

By a mild solution of (3.1), we mean a function $u \in C\left(\mathbb{R} ; L^{2}\right)$ satisfying the integral equation in $L^{2}$ associated with (3.1).

We state the results corresponding to Lemma 1.

Lemma 2. Let $\phi \in \sum$. Then, there exists a unique mild solution $u \in$ $C\left(\mathbb{R} ; \sum\right)$ of $(3.1)$ satisfying

$$
\|u(t)\|_{2}=\|\phi\|_{2}
$$

and

$$
\|\nabla u(t)\|_{2}^{2}+P(u(t))=\|\nabla \phi\|_{2}^{2}+P(\phi)
$$

for $t \in \mathbb{R}$, where 


$$
P(\phi)=\frac{1}{2} \int_{\boldsymbol{R}^{n}} \int_{\boldsymbol{R}^{n}} \frac{|\phi(x)|^{2}|\phi(y)|^{2}}{|x-y|^{\gamma}} d x d y .
$$

Furthermore, $u$ satisfies

$$
\begin{aligned}
&\|(x+2 i t \nabla) u(t)\|_{2}^{2}+4 t^{2} P(u(t)) \\
&=\|(x+2 i s \nabla) u(s)\|_{2}^{2}+4 s^{2} P(u(s)) \\
&+4(2-\gamma) \int_{s}^{t} \tau P(u(\tau)) d \tau, \quad t, s \in \boldsymbol{R}, \\
& P(u(t)) \leq C\left(n, \gamma,\|\phi\|_{\Sigma}\right) \cdot(1+|t|)^{-\gamma}, \quad t \in \boldsymbol{R},
\end{aligned}
$$

and

$$
\|(x+2 i t \nabla) u(t)\|_{2} \leq C\left(n, \gamma,\|\phi\|_{\Sigma}\right) \cdot(1+|t|)^{b(\gamma)}, \quad t \in \boldsymbol{R},
$$

where $b(r)=1-r / 2$, if $0<r \leq 4 / 3(n \geq 2)$ or $0<r<1 \quad(n=1)$, and $b(r)=0$ if $4 / 3<r<\min (4, n)(n \geq 2)$.

Proof. For the case $4 / 3<r<\min (4, n)$, see [4], [5] and [8]. For the cases $0<r \leq 4 / 3(n \geq 2)$ and $0<r<1(n=1)$, see Appendix in $\S 5$.

Q.E.D.

Since we have Lemma 2, in the same way as in the proof of Theorem 1 we obtain:

Theorem 2. Let $\phi \in \Sigma \backslash\{0\}$ and let $u \in C(\boldsymbol{R} ; \Sigma)$ be the solution given by Lemma 2. Then for any

$$
k>k_{1}=2\left(\|\nabla \phi\|_{2}^{2}+P(\phi)\right)^{1 / 2} /\|\phi\|_{2},
$$

we have

$$
\liminf _{t \rightarrow \pm \infty} \int_{|x|<k|t|}|u(t, x)|^{2} d x>0
$$

Remark 3. When $n=3$ and $r=1$, Glassey [6] has proved that for any $R>0$,

$$
\lim _{t \rightarrow \pm \infty} \int_{|x|<R}|u(t, x)|^{2} d x=0 .
$$

Since we easily obtain $L^{p}$-decay $(2<p<\alpha(n)+1)$ estimates by applying the Gagliardo-Nirenberg inequality to (3.7), we find that (3.9) holds when $n \geq 1$ and $0<r<\min (4, n)$.

Corollary 2. Under the assumptions of Theorem 2, the unique mild solution $u \in C(\boldsymbol{R} ; \Sigma)$ has the estimate 


$$
\liminf _{t \rightarrow \pm \infty}|t|^{n / 2-n / q}|| u(t) \|_{L^{q}(|x|<k|t|)}>0
$$

for any $k>k_{1}$ and $q \in[2, \infty]$.

Remark 4. From the Theorem 3.1 in [8] and the same argument as in [12], we have the following assertion:

Suppose $n \geq 2$ and $1<\gamma<\min (4, n)$. Assume $\phi \in \Sigma \backslash\{0\}$. Let $u \in \mathbb{C}(\mathbb{R} ; \Sigma)$ be the solution given by Lemma 2. Then there exist $0<k^{\prime}<k$ satisfying

$$
\liminf _{t \rightarrow \pm \infty} \int_{k^{\prime}|t|<|x|<k|t|}|u(t, x)|^{2} d x>0
$$

and

$$
\liminf _{t \rightarrow \pm \infty}|t|^{n / 2-n / q}|| u(t) \|_{L^{q}\left(k^{\prime}|t|<|x|<k|t|\right)}>0
$$

for any $q \in[2, \infty]$.

Corollary 3. Under the assumptions of Theorem 2, $P(u(t))$ has the estimate

$$
\liminf _{t \rightarrow \pm \infty}|t|^{\gamma} P(u(t))>0 \text {. }
$$

Proof. We first prove (3.10) in the case $1 \leq r<\min (4, n)$. Let $k>k_{1}$. Then, $|x|,|y|<k|t|$ implies $|x-y|^{\gamma}<(2 k|t|)^{\gamma}$. Consequently,

$$
P(u(t)) \geq \frac{1}{2}\left(\frac{1}{2 k|t|}\right)^{\gamma}\left(\int_{|x|<k|t|}|u(t, x)|^{2} d x\right)^{2}
$$

from which (3.10) follows.

We next assume $0<r<1$. Let $k>2 k_{1}$ and $k|t|>1$. We estimate $P(u(t)$ ) from below as follows:

$$
\begin{aligned}
& P(u(t)) \\
& \geq \frac{1}{2} \iint_{1<|x-y|<k|t|} \frac{|u(t, x)|^{2}|u(t, y)|^{2}}{|x-y|^{\gamma}} d x d y \\
& \quad+\frac{1}{2} \iint_{|x-y|<1} \frac{|u(t, x)|^{2}|u(t, y)|^{2}}{|x-y|^{\gamma}} d x d y \\
& \geq \frac{1}{2}\left(\frac{1}{k|t|}\right)^{\gamma} \iint_{|x-y|<k|t|}|u(t, x)|^{2}|u(t, y)|^{2} d x d y \\
& \geq \frac{1}{2}\left(\frac{1}{k|t|}\right)^{\gamma}\left(\int_{|x|<k|t| / 2}|u(t, x)|^{2} d x\right)^{2} .
\end{aligned}
$$

Since $k / 2>k_{1}$, we obtain (3.10) for $0<r<1$.

Q.E.D. 
Remark 5. (3.6) and (3.10) completely characterize the large time behavior of the so-called direct potential energy $P(u(t))$.

\section{§ 4. Limear Schröđinger Equations with Long-range Perturbation}

In this section we freely use the operator theoretic language (see, e.g., [9] and [14]). We consider the symmetric form

$$
h=h_{0}+h_{1} \text { (as a form sum), }
$$

where $h_{0}$ is defined as $h_{0}[\phi, \psi]=(\nabla \phi, \nabla \psi)$ with form domain $Q\left(h_{0}\right)=H^{1}\left(\mathbb{R}^{n}\right)$ and $h_{1}$ is assumed to be a closed symmetric form relatively bounded with $h_{0}$ bounded less than one.

By the KLMN theorem [14] we see that $h$ is a lower-semibounded closed symmetric form with domain $Q(h)=Q\left(h_{0}\right)$ and that $h$ has a unique self-adjoint operator $H$ with domain $D(H)$ satisfying

$$
D(H) \subset Q(h)
$$

and

$$
\begin{aligned}
& (H \psi, \phi)=h[\psi, \phi] \\
& \quad=(\nabla \psi, \nabla \phi)+h_{1}[\psi, \phi], \quad \text { for } \quad \psi \in D(H) \text { and } \phi \in Q(h) .
\end{aligned}
$$

Moreover, for some $j>0$,

$$
\mathcal{Q}(h)=D\left((H+j)^{1 / 2}\right)
$$

and

$$
h[\psi, \phi]=\left((H+j)^{1 / 2} \psi,(H+j)^{1 / 2} \phi\right)-j(\psi, \phi), \psi, \phi \in Q(h) .
$$

Thus, we conclude by the closed graph theorem that $\left(H_{0}+j\right)^{1 / 2}(H+j)^{-1 / 2}$ is a bounded operator defined on $L^{2}$.

We need the following lemma.

Lemma 3. Let $H$ be as above. Then we have:

(1) $e^{-i t H}$ maps $H^{1}\left(\mathbb{R}^{n}\right)$ into $H^{1}\left(\mathbb{R}^{n}\right)$ continuously and furthermore, there exists a constant $a>0$ such that

$$
\left\|e^{-i t H} \phi\right\|_{H^{1}\left(\boldsymbol{R}^{n}\right)} \leq a\|\phi\|_{H^{1}\left(\boldsymbol{R}^{n}\right)}, \quad(t, \phi) \in \mathbb{R} \times H^{1}\left(\mathbb{R}^{n}\right) .
$$

(2) $e^{-i t H}$ maps $\sum$ into $\sum$ conitinuously and furthermore, there exists a constant $b>0$ such that

$$
\left\|e^{-i t H} \phi\right\|_{\Sigma} \leq b(1+|t|)\|\phi\|_{\Sigma}, \quad(t, \phi) \in \mathbb{R} \times \Sigma .
$$




$$
h[\phi, \phi]=h\left[e^{-i t H} \phi, e^{-i t H} \phi\right], \quad(t, \phi) \in \mathbb{R} \times H^{1}\left(\mathbb{R}^{n}\right) .
$$

Proof: (1) and (2) have been proved by Radin and Simon [13]. We prove (3). Let $\phi \in H^{1}\left(\mathbb{R}^{n}\right)=Q\left(h_{0}\right)=Q(h)$ and fix $t \in \mathbb{R}$. We set $R_{\lambda}=$ $i \lambda(H+i \lambda)^{-1}$ for $\lambda>0$. It follows that

$$
R_{\lambda} e^{-i t H} \phi, \quad R_{\lambda} \phi \in D(H)
$$

and that

$$
\begin{aligned}
& h\left[R_{\lambda} e^{-i t H} \phi, R_{\lambda} e^{-i t H} \phi\right]=\left(H R_{\lambda} e^{-i t H} \phi, R_{\lambda} e^{-i t H} \phi\right) \\
& \quad=\left(H e^{-i t H} R_{\lambda} \phi, e^{-i t H} R_{\lambda} \phi\right)=\left(H R_{\lambda} \phi, R_{\lambda} \phi\right) \\
& \quad=h\left[R_{\lambda} \phi, R_{\lambda} \phi\right] .
\end{aligned}
$$

Since $h$ is a lower-semibounded closed form, we have the assertion if we prove that for any $\psi \in H^{1}\left(\mathbb{R}^{n}\right),\left\{h\left[R_{\lambda} \psi, R_{\lambda} \psi\right] ; \lambda>0\right\}$ is a Cauchy sequence in $\mathbb{R}$. From the assumption on $h_{1}$, it suffices to show

$$
\left\|V\left(R_{\lambda} \psi-R_{\mu} \psi\right)\right\|_{2} \rightarrow 0 \quad \text { as } \quad \lambda, \mu \rightarrow \infty .
$$

We now do this. Let $j>0$ be as in (4.4)-(4.5). Then,

$$
\begin{aligned}
& \left\|\nabla\left(R_{\lambda} \psi-R_{\mu} \psi\right)\right\|_{2}^{2} \\
& \quad=\left\|\left(H_{0}+j\right)^{1 / 2}\left(R_{\lambda} \psi-R_{\mu} \psi\right)\right\|_{2}^{2}-j\left\|R_{\lambda} \psi-R_{\mu} \psi\right\|_{2}^{2} \\
& \quad \leq\left\|\left(H_{0}+j\right)^{1 / 2}(H+j)^{-1 / 2}\right\|_{\mathcal{L}\left(L^{2}\right)^{\circ}}^{\circ}\left\|(H+j)^{1 / 2}\left(R_{\lambda} \psi-R_{\mu} \psi\right)\right\|_{2}^{2} \\
& \quad=\left\|\left(H_{0}+j\right)^{1 / 2}(H+j)^{-1 / 2}\right\|_{\mathcal{L}\left(L^{2}\right)^{\circ}}\left\|\left(R_{\lambda}-R_{\mu}\right)(H+j)^{1 / 2} \psi\right\|_{2}^{2} \rightarrow 0 \\
& \quad(\lambda, \mu \rightarrow \infty),
\end{aligned}
$$

which proves our claim.

Q.E.D.

We now state the assumption on $H$.

(H) For any $\phi \in \sum \cap \mathcal{H}_{\text {cont }}(H)$, we have

$$
\lim _{t \rightarrow \pm \infty}\left\|\left(\frac{x}{2 i t}+\nabla\right) e^{-i t H} \phi\right\|_{2}=0 .
$$

By virtue of Lemma 3, the conditions given by Enss [2] are sufficient for (4.6) to hold. They cover the case where $h_{1}$ is obtained by the following perturbation $V$ :

$V$ is decomposable as $V=V_{s}+V_{l}$, where $V_{s}$ is a short-range potential and $V_{l}$ is a multiplication operator by a continuously differentiable real-valued function $V_{l}$ satisfying

$$
V_{l}(x), x \cdot \nabla V_{l}(x) \rightarrow 0 \quad \text { as } \quad|x| \rightarrow+\infty .
$$


Theorem 3. Let $H$ be as above. Assume that $(H)$ holds. Let $\phi \in$ $\left(\Sigma \cap \mathcal{H}_{\text {cont }}(H)\right) \backslash\{0\}$. Then for any

$$
k>k_{2}:=2(h[\phi, \phi])^{1 / 2} /\|\phi\|_{2},
$$

we have

$$
\liminf _{t \rightarrow \pm \infty} \int_{|x|<k|t|}\left|\left(e^{-i t H} \phi\right)(x)\right|^{2} d x>0
$$

If in addition, there exist $t_{0}>0$ and $q \in[2, \infty]$ such that

$$
e^{-i t H} \phi \in L_{\mathrm{loc}}^{q}\left(\mathbb{R}^{n}\right), \quad|t| \geq t_{0},
$$

then,

$$
\liminf _{t \rightarrow \pm \infty}|t|^{n / 2-n / q}|| e^{-i t H} \phi \|_{L^{q}(|x|<k|t|)}>0 .
$$

Proof. Proof is immediate since we have Lemma 3-3) and (H). Q.E.D.

\section{§5. Appendix}

In this appendix, we prove Lemma 2 in the cases $0<r \leq 4 / 3(n \geq 2)$ and $0<r<1(n=1)$. For $T>0$, we introduce the following Banach psace $B_{T}$ by

$$
B_{T}=C\left([-T, T] ; \sum\right) \text { with the norm }\|u\|_{B_{T}}=\sup _{|t| \leq T}\left\|e^{i t H_{0}} u(t)\right\|_{\Sigma}
$$

and the closed ball $B_{T}(\rho)(\rho>0)$ by

$$
B_{T}(\rho)=\left\{u \in B_{T} ;\|u\|_{B_{T}} \leq \rho\right\} .
$$

Note that $\|\cdot\|_{B T}$ is an equivalent norm to the usual norm on $C([-T, T] ; \Sigma)$.

We are now in a position to complete the proof of Lemma 2.

Proof of Lemma 2 in the cases $0<r \leq 4 / 3(n \geq 2)$ and $0<r<1 \quad(n=1)$.

Let $w \in B_{T}(\rho)$. We define $S w$ by

$$
(S w)(t)=e^{-i t H_{0}} \phi-i \int_{0}^{t} e^{-i(t-\tau) H_{0}}\left(\left(V *|w|^{2}\right) w(\tau)\right) d \tau, \quad|t| \leq T .
$$

In the same way as in the proof of Theorem 4.1 in [8] we get

$$
\left\|e^{i \tau H_{0}}\left(\left(V *|w|^{2}\right) w(\tau)\right)\right\|_{\Sigma} \leq C(n, r) g(\tau)\left\|e^{i \tau H_{0}} w(\tau)\right\|_{\Sigma}, \quad|\tau| \leq T,
$$

where $g(\tau)=\|w(\tau)\|_{r}^{2}+\|w(\tau)\|_{r+\varepsilon}^{2}+\|w(\tau)\|_{r-\varepsilon}^{2}$ with sufficiently small $\varepsilon>0$ and $r=2 r i /(n-r)$.

For $0<r<\min (2, n)$, we have by the Gagliardo-Nirenberg inequality 


$$
\|w(t)\|_{r} \leq C(n, \gamma)\|w(t)\|_{2}^{1-\gamma / 2}\|\nabla w(t)\|_{2}^{\gamma / 2},
$$

and

$(5.4)_{ \pm}$

$$
\|w(t)\|_{r \pm \varepsilon} \leq C(n, \gamma)\|w(t)\|_{2}^{1-\gamma( \pm \varepsilon) / 2}\|\nabla w(t)\|_{2}^{\gamma( \pm \varepsilon)},
$$

where

$$
r( \pm \varepsilon)=\left(r \pm \frac{n}{2}\left(1-\frac{r}{n}\right) \varepsilon\right) /\left(2 \pm\left(1-\frac{r}{n}\right) \varepsilon\right)
$$

(5.2) $-(5.4)_{ \pm}$imply

$$
\begin{aligned}
& \left\|e^{i \tau H_{0}}\left(\left(V *|w|^{2}\right) w(\tau)\right)\right\|_{\Sigma} \\
& \quad \leq C(n, \gamma) \rho^{2} \cdot \sup _{|t| \leq T}\left\|e^{i \tau H_{0}} w(\tau)\right\|_{\Sigma} \\
& \quad \leq C(n, \gamma) \rho^{3}, \quad t \in[-T, T],
\end{aligned}
$$

from which it follows that

$$
S w \in C([-T, T] ; \Sigma)
$$

and

$$
\begin{aligned}
& \left\|e^{i t H_{0}}(S w)(t)\right\|_{\Sigma} \\
& \quad \leq\|\phi\|_{\Sigma}+\left\|\int_{0}^{t} e^{i \tau H_{0}}\left(\left(V *|w|^{2}\right) w(\tau)\right) d \tau\right\|_{\Sigma} \\
& \quad \leq\|\phi\|_{\Sigma}+C(n, \gamma) \rho^{3}|t|, \quad t \in[-T, T] .
\end{aligned}
$$

This gives

$$
\|S w\|_{B_{T}} \leq\|\phi\|_{\Sigma}+C(n, r) \rho^{3} T .
$$

We also have for $w_{1}^{\prime}, w_{2} \in B_{T}$ that

$$
\left\|S w_{1}-S w_{2}\right\|_{B_{T}} \leq C(n, \gamma) \rho^{2} T\left\|w_{1}-w_{2}\right\|_{B_{T}} .
$$

If $\rho$ and $T$ are chosen to satisfy

$$
\rho \geq 2\|\phi\|_{\Sigma} \quad \text { and } \quad T \leq 1 /\left(2 C(n, \gamma) \rho^{2}\right),
$$

then (5.7) and (5.8) allows us to conclude that $S$ is a contraction mapping from $B_{T}(\rho)$ into itself. This implies that there exists a unique mild solution $u \in$ $B_{T}(\rho)$ for sufficiently small $T>0$. Furthermore, along the line of the argument of Ginibre and Velo [4] it is easily verified that $u$ satisfies (3.2), (3.3) and (3.5) for any $t, s \in[-T, T]$. Then, by virtue of (3.2), (3.3), (3.5) and the Gagliardo-Nirenberg inequality we have

$$
\|u(t)\|_{2},\|\nabla u(t)\|_{2} \leq C(n, \gamma,\|\phi\| \Sigma)
$$


and

$$
\left\|x e^{i t H_{0}} u(t)\right\|_{2} \leq C\left(n, \gamma,\|\phi\|_{\Sigma}\right) \cdot(1+|t|)
$$

for any $t \in[-T, T]$. From (5.9) and (5.10) it follows that for any $T>0$ there exists a unique mild solution $u \in B_{T}$ of (3.1) satisfying (3.2) and (3.3) for any $t \in \mathbb{R}$ and that $u$ satisfies (3.5) for any $t, s \in \boldsymbol{R}$. Therefore we have $u \in C(\boldsymbol{R} ; \Sigma)$. In the same fashion as in the proof of (5.9) and (5.10) in [8], we observe that $u$ satisfies (3.6) and (3.7) with $b(r)$ replaced by $1-r / 2$. This completes the proof.

Q.E.D.

\section{References}

[1] Barab, J.E., Nonexistence of asymptotic free solutions for a nonlinear Schrödinger equation, J. Math. Phys., 25 (1984), 3270-3273.

[2] Enss, V., Asymptotic observables on scattering states, Comm. Math. Phys., 89 (1983), 245-268.

[3] Ginibre, J. and Velo, G., On a class of nonlinear Schrödinger equations I, II, $J$. Funct. Anal., 32 (1979), 1-32, 33-71.

[4] - On a class of nonlinear Schrödinger equations with non local interaction, Math. Z., 170 (1980), 109-136.

[5] - Sur une équation de Schrödinger non lincáire avec interaction non locale, in "nonlinear Partial Differential Equations and Their Applications," College de France Seminar, Vol. II, Pitman, Boston, 1981.

[6] Glassey, R.T., Asymptotic behavior of solutions to certain nonlinear SchrödingerHartree equations. Comm. Math. Phys., 53 (1977), 9-18.

[7] Hayashi, N. and Tsutsumi, M., $L^{\infty}\left(\mathbb{R}^{n}\right)$-decay of classical solutions for nonlinear Schrödinger equations, Proceedings of the Royal Society of Edinburgh, 104A (1986), 309-327.

[8] Hayashi, N. and Tsutsumi, Y., Scattering theory for Hartree type equations, Ann. Inst. Henri Poincaré, Physique théorique, 46 (1987), 187-213.

[9] Kato, T., "Perturbation Theory for Linear Operators." Second Edition, SpringerVerlag, Berlin, Heiderberg. New York, 1976.

[10] Lin, J.E. and Strauss, W.A., Decay and scattering of solutions of a nonlinear Schrödinger equation, J. Funct. Anal., 30 (1978), 245-263.

[11] Ozawa, T., New $L^{p}$-estimates for solutions to the Schrödinger equations and time asymptotic behavior of observables, in Publ. RIMS, Kyoto Univ., 25 (1989), 521-577.

[12] - Lower $L^{p}$-bounds for scattering solutions of the Schrödinger equations, Publ. RIMS, Kyoto Univ., 25 (1989), 579-586.

[13] Radin, C. and Simon, B., Invariant domains for the time-dependent Schrödinger equation, J. Differential Equations, 29 (1978), 289-296.

[14] Reed, M. and Simon, B., "Methods of Modern Marhematical Physics," I: Functional Analysis (1972), II: Fourier Analysis, Self-adjointness (1975), Academic Press, New York.

[15] Tsutsumi, Y., "Global existence and asymptotic behavior of nonlinear Schrödinger equations," Doctoral Thesis, University of Tokyo. 
